\title{
Right orders in full linear rings
}

\section{Kovin C. O'Moara}

One way to generalize the Goldie Theorems [3] on prime and semi-prime rings is by studying right orders in rings which have zero (Jacobson) radical but for which the finiteness requirement is relaxed, for example, regular right self-injective rings. The rings studied in this thesis, right orders in a full linear ring $Q=\operatorname{hom}_{D}(V, V), V$ a right vector space over a division ring $D$, fall into this category. The problem of characterizing such rings was posed by Faith [1], problem 12, p. 129. Since a regular right self-injective ring whose socle is large as a one-sided ideal is a direct product of full linear rings, it is clear how one could approach the study of right orders in these rings from our study.

In the sequel, $Q$ denotes a full linear ring. For a right ideal $I$ of $Q$, we denote the uniform dimension of $I_{Q}$ by $\operatorname{dim} I$. If $R$ is a subring of a ring $S$, then $S$ is a right quotient ring of $R$ (respectively $S$ is right intrinsic over $R$ ) if $A \cap R \neq 0$ for each nonzero submodule $A$ of $S_{R}$ (respectively right ideal of $S$ ). If $S$ has an identity, then we say that $R$ is a right order in $S$ if

(i) regular elements of $R$ have (two-sided) inverses in $S$, and

(ii) the elements of $S$ can be expressed in the form $b c^{-1}$, where $b, c \in R$ and $c$ is a unit of $S$.

Received 29 December 1971. Thesis submitted to the University of Canterbury, September 1971. Degree approved, December 1971. Supervisor: Dr Robert L. Kruse. 
We now outline the contents of the thesis. Chapter I is introductory. One of the principal results in Chapter II is that right orders in full linear rings of countable dimension must be prime rings, whereas in the uncountable case this need not be so. A class of non-prime right orders is exhibited in the following theorem.

THEOREM. Suppose $e_{1}, \ldots, e_{n}$ are nonzero orthogonal idempotents of $Q$ with $e_{1}+\ldots+e_{n}=1, n \geq 2$. Let $P=\sum_{i \leq j} e_{i} Q e_{j}$. Then $P$ is a right order in $Q$ if and only if

$$
\operatorname{dime}_{1} Q>\operatorname{dime}_{i} Q \text { for } i=2, \ldots, n
$$

and

$$
\text { dime }_{n} Q \geq \kappa_{0} \text {. }
$$

If $R$ is a right order in $Q$ and $R$ contains a nilpotent ideal of index $n \geq 2$, then $R$ appears as a subring of $P$ for some $P$ given as above.

COROLLARY. If $R$ is both a left and right order in $Q$, then $R$ is a prime ring.

Chapter III is a study of intrinsic extensions of prime rings. This study is required by the condition that regular elements of a right order $R$ in $Q$ be units in $Q$, since this actually implies $Q$ is left intrinsic over $R$ when dimQ is infinite. Thus, in marked contrast to prime right Goldie rings, a right order $R$ in an infinite dimensional full linear ring has the property that closed (= complement) right ideals of $R$ are right annihilator ideals. The principal result on intrinsic extensions says that if $S$ is a prime ring with zero left singular ideal, but not an integral domain, and $S$ contains uniform left ideals, then $S$ is a left quotient ring of each prime ring over which it is left intrinsic. Thus when dimQ is countably infinite, a right order in $Q$ must have $Q$ also as a left quotient ring.

The main goal of Chapter IV is finding suitable conditions to ensure that a ring $R$ will have a full linear ring as its left-flat epimorphic hull (see [2]). To this end, two conditions are introduced: condition (A) requiring closed right ideals of $R$ to be essential extensions of finitely 
generated right ideals, and the existence of a "reducing pair" of elements. The latter means a pair $(\beta, \gamma)$ for which $\beta R, \gamma R$ and $\beta^{p}+\gamma^{r}$ are large right ideals of $R$. Taken together, these two conditions on a ring $R$ having $Q$ as a right quotient ring imply that for each $x \in Q$ there exists $c \in R$ such that $c$ has a right inverse in $Q$ and $x c \in R$. Ample evidence is produced to show that reducing pairs for infinite dimensional irreducible rings $R$ play a similar role to primeness for finite dimensional irreducible $R$, in so far as determining when $R$ is a right order in a full linear ring.

The final chapter contains, among other things, two internal characterizations of a right order $R$ in an infinite dimensional full linear ring. One of these says :

THEOREM. A ring $R$ is a right order in a full linear ring of dimension $K \geq \aleph_{0}$ if and only if the following conditions are satisfied:

(i) $R$ is a (Johnson) irreducible ring containing uniform right ideals and $\operatorname{dim} R_{R}=\aleph$;

(ii) the closed right ideals of $R$ are right annihilator ideals and each such right ideal is an essential extension of a finitely generated right ideal;

(iii) $R$ possesses a reducing pair of elements;

(iv) for each $a \in R$ with $a^{2}=0, a R$ contains regular elements of $R$.

\section{References}

[1] Carl Faith, Lectures on injective modules and quotient rings (Lecture Notes in Mathematics, 49. Springer-Verlag, Berlin, Heidelberg, New York, 1967).

[2] George D. Findlay, "Flat epimorphic extensions of rings", Math. 2. 118 (1970), 281-288.

[3] A.W. Goldie, "Semi-prime rings with maximum condition", Proc. London Math. Soc. (3) 10 (1960), 201-220. 